\title{
Erratum to Free Arrangement and Rhombic Tilings
}

\author{
P. H. Edelman and V. Reiner \\ School of Mathematics, University of Minnesota, \\ Minneapolis, MN 55455, USA \\ edelman@math.umn.edu \\ reiner@math.umn.edu
}

The authors are indebted to Stefan Felsner for pointing out an error in the proof of Lemma 5.2 [ER], which invalidates both the lemma and the proof of Theorem 5.1. Specifically, it is asserted in the proof of Lemma 5.2 that the binary relation $>$ has no directed cycles, but in fact, for $n=6$ and $T$ the tiling having noninversions $\{123,456\}$, it can be checked that this relation has the following directed cycle:

$$
256>235>125>245>256 .
$$

The faulty assertion was based on an incomplete case analysis. A missing case was brought to our attention by Felsner.

As a consequence, the question of whether the higher Bruhat order $B(n, 2)$ defined by Manin and Schechtman [MS] (via single-step inclusion of inversion sets) coincides with the order $B_{\subseteq}(n, 2)$ defined by Ziegler [Z] (via inclusion of the inversion sets) remains unresolved. Also, the partial order $H B\left(r_{1}, r_{2}, \ldots, r_{l}\right)$ introduced in this paper is strictly speaking a generalization of $B_{\subseteq}(n, 2)$ and not of $B(n, 2)$.

\section{References}

[ER] P. H. Edelman and V. Reiner, Free arrangements and rhombic tilings, Discrete Comput. Geom. 15 (1996), 307-340.

[MS] Y. I. Manin and V. V. Schechtman, Arrangements of hyperplanes, higher braid groups, and higher Bruhat orders, Adv. Stud. in Pure Math. 17 (1989), 289-308.

[Z] G. Ziegler, Higher Bruhat orders and cyclic hyperplane arrangements, Topology 32 (1993), 259-279.

Received August 30, 1996. 